\title{
Energy Exchange Coordination of Off-Grid Charging Stations with Vehicular Energy Network
}

\author{
James J.Q. Yu, Albert Y.S. Lam, and Siew-Chong Tan \\ Department of Electrical and Electronic Engineering \\ The University of Hong Kong \\ Pokfulam Road, Hong Kong \\ Email: \{jqyu, ayslam, sctan\}@eee.hku.hk
}

\begin{abstract}
Renewable energy and electric vehicles (EVs) are two key developments of smart grid. To support the increasing charging demands of EVs, more charging stations need to be constructed. Some charging stations are located in remote areas and they mainly rely on renewable generations for energy provisioning. Due to dissimilar self-generations and local energy demands at different locations, it is beneficial to share the energy among themselves for a sustainable eco-system. Powered by the smart city vision that revolutionizes the energy and transportation sectors, vehicular energy network (VEN) is developed, which can distribute energy across a geographical area by means of EVs. In this paper, we formulate an optimization problem to optimally coordinate energy exchange among renewable-enabled or off-grid charging stations based on VEN. We also propose a heuristic to develop some high-quality energy tracks for energy exchange, which can significantly reduce the computational time. We evaluate the energy exchange performance on some random networks of various sizes. Simulation results demonstrate that the proposed design can effectively deliver excessive energy over VEN and the proposed heuristic can drastically reduce the computational time without significantly undermining the performance.
\end{abstract}

\section{INTRODUCTION}

The smart grid facilitates the development of many advanced energy control technologies, in which renewable energy and electric vehicles (EVs) take crucial roles. They alleviate our reliance on fossil fuels and to foster sustainability. While the widespread renewable energy sources facilitate the shift of generations from central plants toward distributed energy resources, EVs can serve as flexible energy repositories for the grid. Many studies investigate various smart grid applications utilizing EVs, vehicle-to-grid technologies [1], and renewables [2], [3]. [4] and [5] provide comprehensive surveys of renewable energy and $\mathrm{EV}$ research.

Electrical energy is essential to powering EVs and the gradual adoption of EVs advocates extensive constructions of EV charging stations [6]. However, for some reasons, certain charging stations need to be located at remote areas. Their connections to the main grid may not always be physically possible or lots of efforts are required to bring them online. To acquire energy for EV charging, many charging stations are equipped with self-generation facilities, such as photovoltaic panels [7] and wind turbines [8]. This can not only address energy provisioning but also increase renewable energy penetration, constituting a greener transportation system.
Even for the grid-connected charging stations, energy provisioning by the renewables may also bring into another dimension of problems. Due to the intermittency of renewables, extra measures are definitely required to ensure the stability of power system (see [9] for an example). For some charging stations spread over a region, consider that their self renewable energy generations are uneven and their charging demands are highly heterogeneous. It is likely that some of the charging stations are generating excessive energy while the others consume more than their own generations. If there exists an independent energy delivery system which enables energy exchange among these charging stations, we can create a sustainable eco-system of charging stations and utilize the renewable energy more effectively without impairing the power grid stability.

Recently the vehicular energy network (VEN) has been developed, which is capable of transmitting energy over a geographical region effectively via EVs [10]. VEN is basically constructed over a road network, which is well-established to cover most areas with human activities. At some locations along the roads, wireless charging-discharging facilities are installed with energy storage and they allow the passing EVs to get charged or discharged over the air on the move. While traversing the road network based on their own schedules, with proper charging and discharging, EVs can carry energy to different locations in the network. Even if each EV carries a very small energy packet each time (which does not harm the battery much [11]), the aggregated energy flows in VEN can still be substantial to support the energy exchange among different locations [10]. There are also other studies dedicated to VEN routing strategies for improving its energy delivery efficiency [12], [13]. Therefore, VEN is a promising infrastructure for energy delivery in an effective and fully controllable manner [10].

In this paper, we study the coordination of charging stations for energy exchange with VEN. Without loss of generality, we assume off-grid charging station 11 such that they can operate as an independent system without relying on the grid. To facilitate the energy exchange, we establish energy tracks among charging stations over VEN. We formulate a multitrack coordination optimization problem for energy exchange

\footnotetext{
${ }^{1}$ Here we can also cover the grid-connected charging stations. Our assumption can allow them to operate independently most of the time and to acquire power from the grid only when necessary.
} 


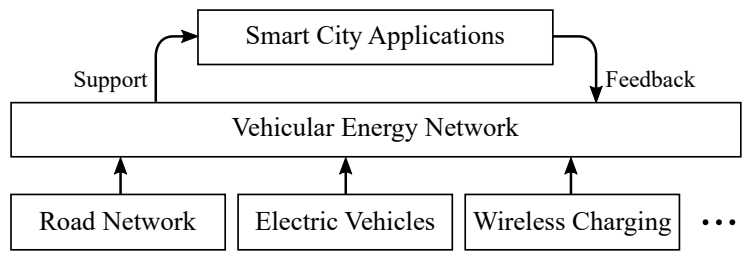

Fig. 1. Schematic structure of VEN and smart city applications.

between the charging stations systematically. In addition, as the number of energy tracks is enormous, we propose a track selecting heuristic to strategically develop the energy tracks for the optimization model. This can drastically reduce the computational time while maintaining the system performance.

The rest of this paper is organized as follows. In Section II, we briefly introduce the system model of VEN and we formulate the multi-track energy exchange problem in Section III. In Section IV, we propose the track selecting heuristic and we present case studies to demonstrate the efficacy of the optimization and proposed heuristic in Section V. Finally, this work is concluded in Section VI.

\section{SyStem Model}

This paper demonstrates an application of VEN, in which we study how to exchange energy among some charging stations. In general, we can understand the role of VEN with Fig. 1. A road network, a fleet of EVs, some wireless charging facilities, and other technologies constitute VEN, which in turn provides energy delivery services for the applications built on top of it. This layered structure can abstract many design considerations of VEN so that we can focus on the details of the application.

\section{A. Vehicular Energy Network}

We basically follow [10] to define VEN, which is composed of an underlying road network and an overlying energy network:

1) Road Network: EVs travel in the road network, which is modeled by a direct graph $\mathcal{G}(\mathcal{N}, \mathcal{A})$, where $\mathcal{N}$ and $\mathcal{A}$ are road intersections and segments, respectively. Consider that all $n \in \mathcal{N}$ are equipped with (dis)charging facilities and they are called energy points in the sequel. They can be further classified into three types: $\mathcal{N}^{\mathrm{S}}, \mathcal{N}^{\mathrm{D}}$, and $\mathcal{N}^{\mathrm{R}} \cdot \mathcal{N}^{\mathrm{S}}$ and $\mathcal{N}^{\mathrm{D}}$ refer to the energy sources and destinations, which are those charging stations with excessive and deficient energy, respectively. $\mathcal{N}^{\mathrm{R}}$ are the routing points for energy exchange between vehicles only. $\bar{G}_{s} \geq 0$ denotes the maximum amount of energy available to be shared at $s \in \mathcal{N}^{\mathrm{S}}$ and $\bar{C}_{d} \geq 0$ represents the minimum amount of energy needed at $d \in \mathcal{N}^{\mathrm{D}}$. Each $a=\left(n_{a}^{\mathrm{fr}}, n_{a}^{\mathrm{to}}\right) \in \mathcal{A}$ has a starting point $n_{a}^{\mathrm{fr}}$ and an ending point $n_{a}^{\text {to }}$. Moreover, as the number of EVs driving on $a$ in each time slot is finite, each segment $a$ has an energy transmission capacity, denoted by $\bar{W}_{a}$.



Fig. 2. An example of multiple energy paths for an energy source-destination pair.

2) Energy Network: The energy network is an abstract network built on top of the road network. Each pair of energy source and destination are connected by a set of energy paths [10], each of which is composed of one or multiple road segments in $\mathcal{G}(\mathcal{N}, \mathcal{A})$. For the same energy source and destination, it is possible to construct different energy paths with the same set of vehicular paths of subtle different configurations [10]. However, these energy paths may complicate the application designed over VEN. Hence, in this paper, we simplify the design by aggregating those energy paths with the same source-destination pair and constituting vehicular routes into one single energy track so that we can focus on how to share the energy among charging stations. Once the energy tracks have been developed for the application, we can construct the corresponding energy paths in VEN to realize the energy tracks based on [12] and [14].

For each pair of $s \in \mathcal{N}^{\mathrm{S}}$ and $d \in \mathcal{N}^{\mathrm{D}}$, we can construct the set of all possible energy tracks, denoted by $\mathcal{P}(s, d)$. The energy generated at $s$ can be transmitted to $d$ using one or multiple of these tracks. Each $p_{i}(s, d) \in \mathcal{P}(s, d)$ is represented by its constructing segments $\left\langle a_{1}, a_{2}, \cdots, a_{j}, \cdots, a_{J_{i}(s, d)}\right\rangle$, where $a_{j} \in \mathcal{A}$ is the $j$-th segment of $p_{i}(s, d)$, and $J_{i}(s, d)=$ $\left|p_{i}(s, d)\right|$ is the number of total road segments in the track. Therefore, we have $s=n_{a_{1}}^{\mathrm{fr}}, d=n_{a_{J}}^{\mathrm{to}}$, and $n_{a_{j-1}}^{\mathrm{to}}=n_{a_{j}}^{\mathrm{fr}}$, for $1<j \leq J_{i}(s, d)$. We also define the actual amount of energy transmitted over $p_{i}(s, d)$ by $E_{i}(s, d)$. Consider the example given in Fig. 2 Nodes $\mathrm{A}$ and $\mathrm{D}$ are an energy source and a destination, respectively, while Nodes $\mathrm{B}$ and $\mathrm{C}$ are routing points. Each road segment is associated with its energy transmission capacity $\bar{W}_{a}$. In this network, there are three energy tracks connecting $A$ and $D$ : $p_{1}(\mathrm{~A}, \mathrm{D})=\langle\langle\mathrm{A}, \mathrm{B}\rangle,\langle\mathrm{B}, \mathrm{D}\rangle\rangle, p_{2}(\mathrm{~A}, \mathrm{D})=\langle\langle\mathrm{A}, \mathrm{C}\rangle,\langle\mathrm{C}, \mathrm{D}\rangle\rangle$, and $p_{3}(\mathrm{~A}, \mathrm{D})=\langle\langle\mathrm{A}, \mathrm{C}\rangle,\langle\mathrm{C}, \mathrm{B}\rangle,\langle\mathrm{B}, \mathrm{D}\rangle\rangle$. The transmittable energy of each track should be no larger than the capacity of any of its constituting segments. Meanwhile, as $p_{1}(\mathrm{~A}, \mathrm{D})$ and $p_{3}(\mathrm{~A}, \mathrm{D})$ share $(\mathrm{B}, \mathrm{D})$, the sum of energy transmitted along these two energy tracks must not exceed the capacity of $(\mathrm{B}, \mathrm{D})$. Therefore, $E_{1}(\mathrm{~A}, \mathrm{D})+E_{3}(\mathrm{~A}, \mathrm{D}) \leq \bar{W}_{\langle\mathrm{B}, \mathrm{D}\rangle}=2$, and similarly, $E_{2}(\mathrm{~A}, \mathrm{D})+E_{3}(\mathrm{~A}, \mathrm{D}) \leq \bar{W}_{\langle\mathrm{A}, \mathrm{C}\rangle}=3$ for sharing $(\mathrm{A}, \mathrm{C})$.

Each energy track also experiences charging-discharging cycles at its energy points resulting in energy loss. Assume that the energy efficiency for each charging-discharging cycle is $H$. Then the energy efficiency $\eta_{i}(s, d)$ for route $p_{i}(s, d)$ is determined by $\eta_{i}(s, d)=H^{J_{i}(s, d)}$. 


\section{B. Operation}

Consider that there is a control center which coordinates the energy exchange among the charging stations. We aim to maximize the utilization of excessive energy generated at the energy sources and minimize the energy loss subject to some energy consumption requirements. We focus on coordinating the energy exchange for a given period of time. We first set up the energy exchange plan among charging stations and then carry out the plan over VEN in the rest of the period.

At the beginning of planning, each charging station evaluates its energy generation and consumption conditions, and reports to the control center. $\bar{G}_{s}$ for each $s \in \mathcal{N}^{s}$ and $\bar{C}_{d}$ for each $d \in \mathcal{N}^{d}$ are determined. The control center then develops the required energy tracks for each source-destination pair and optimizes the amount of energy $E_{i}(s, d)$ for each energy track by solving an optimization problem, which is discussed in Section [III. The result is then distributed to the corresponding energy points and the energy sources then start delivering energy based on $E_{i}(s, d)$ through VEN. Meanwhile, energy may not be available immediately at the energy destinations due to various delays [10], e.g., travel time of vehicles. In fact, energy may first be advanced from the attached energy storage at routing points and the energy destinations if necessary. When the appropriate vehicles arrive at these locations later on, the on-board VEN energy is discharged to the corresponding storage to cover the deficit.

\section{Problem Formulation}

We should optimally distribute the excessive generated energy among the charging stations via the energy tracks. In this section, we formulate the problem as an optimization.

Consider $E_{i}(s, d)$ a control variable, which should be nonnegative by nature, i.e.,

$$
E_{i}(s, d) \geq 0, \forall s \in \mathcal{N}
$$

$E_{i}(s, d)$ should also be limited by the maximum energy generated at all $s \in \mathcal{N}^{\mathrm{S}}$ and confined by the minimum energy drawn from $d \in \mathcal{N}^{\mathrm{D}}$. Thus we have

$$
\begin{array}{r}
\sum_{d \in \mathcal{N}^{\mathrm{D}}} \sum_{i=1}^{I(s, d)} E_{i}(s, d) \leq \bar{G}_{s}, \forall s \in \mathcal{N}^{\mathrm{S}}, \\
\sum_{s \in \mathcal{N}^{\mathrm{S}}} \sum_{i=1}^{I(s, d)} \eta_{i}(s, d) E_{i}(s, d) \geq \bar{C}_{d}, \forall d \in \mathcal{N}^{\mathrm{D}},
\end{array}
$$

where $I(s, d)=|\mathcal{P}(s, d)|$.

As analyzed in Section II-A2, the transmitting energy must not exceed the capacity of the energy track, which gives

$$
\begin{array}{r}
E_{i}(s, d) \leq \bar{W}_{a}, \forall a \in p_{i}(s, d), 1 \leq i \leq I(s, d), \\
\forall s \in \mathcal{N}^{\mathrm{S}}, d \in \mathcal{N}^{\mathrm{D}} .
\end{array}
$$

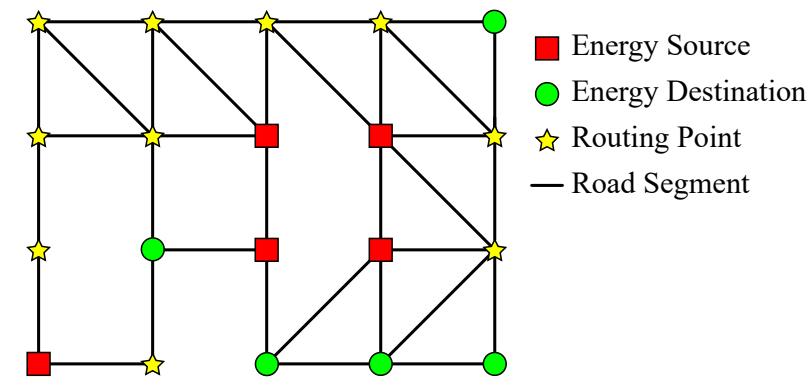

Fig. 3. A randomly generated VEN with two-way interconnects.

It is possible to have multiple energy tracks sharing a road segment. In such case, the total amount of energy transmitted by all these tracks must not exceed its capacity. So we have

$$
\sum_{s \in \mathcal{N}^{\mathrm{S}}} \sum_{d \in \mathcal{N}^{\mathrm{D}}} \sum_{i=1}^{I(s, d)} \hat{E}_{i}^{a}(s, d) \leq \bar{W}_{a}, \forall a \in \mathcal{A},
$$

where

$$
\hat{E}_{i}^{a}(s, d)=\left\{\begin{array}{ll}
E_{i}(s, d) & \text { if } a \in p_{i}(s, d) \\
0 & \text { otherwise }
\end{array}, \forall a \in \mathcal{A}\right.
$$

is the energy contributed by $p_{i}(s, d)$ on road segment $n \in \mathcal{N}$.

Since $E_{i}^{a}(s, d) \geq 0$, we have

$$
\begin{array}{r}
\sum_{s \in \mathcal{N}^{\mathrm{S}}} \sum_{d \in \mathcal{N}^{\mathrm{D}}} \sum_{i=1}^{I(s, d)} \hat{E}_{i}^{a}(s, d) \geq \hat{E}_{i}^{a}(s, d), \forall a \in p_{i}(s, d), \\
1 \leq i \leq I(s, d), \forall s \in \mathcal{N}^{\mathrm{S}}, d \in \mathcal{N}^{\mathrm{D}} .
\end{array}
$$

Combining (5)-77) gives

$$
\begin{array}{r}
E_{i}(s, d)=\hat{E}_{i}^{a}(s, d) \leq \bar{W}_{a}, \forall a \in p_{i}(s, d), 1 \leq i \leq I(s, d), \\
\forall s \in \mathcal{N}^{\mathrm{S}}, d \in \mathcal{N}^{\mathrm{D}} .
\end{array}
$$

In fact, (5) is equivalent to (4) but the former is tighter giving better performance in solving the optimization.

Finally, as mentioned in Section II] we consider the following two objectives:

1) Energy delivery maximization:

$$
\operatorname{maximize} E^{\text {total }}=\sum_{s \in \mathcal{N}^{\mathrm{S}}} \sum_{d \in \mathcal{N}^{\mathrm{D}}} \sum_{i=1}^{I(s, d)} \eta_{i}(s, d) E_{i}(s, d) ;
$$

2) Energy loss minimization:

$$
\operatorname{minimize} E^{\mathrm{loss}}=\sum_{s \in \mathcal{N}^{\mathrm{S}}} \sum_{d \in \mathcal{N}^{\mathrm{D}}} \sum_{i=1}^{I(s, d)}\left[1-\eta_{i}(s, d)\right] E_{i}(s, d) .
$$

These two objectives are optimized individually in this work, subject to constraints introduced by (1), (2), (3), and (5). 


\section{Track Selecting Heuristic}

To formulate Problem (8), we assume full knowledge of all possible energy tracks in the system. However, as shown in [12], the total number of energy paths in VEN grow exponentially with the number of energy points, so as the energy tracks for VEN applications. For the example shown in Fig. 3. a relatively small network with 5 energy sources, 5 energy destinations, and 10 routing points has 296,541 energy tracks. Therefore, modeling and solving the proposed optimization problem can be computationally expensive. To alleviate the computational complexity, in this section we propose a track selecting heuristic to strategically select some "good" energy tracks for optimization.

The heuristic is constructed based on the intuition that energy tracks with fewer charging-discharging cycles are more preferable as they incur less energy loss. Its pseudo-code is presented in Algorithm 11. In this heuristic, the pre-defined parameter $\bar{I}(s, d)$ represents the total number of required energy tracks from $s$ to $d$. This parameter has a direct impact on the resultant problem size and we will analyze its sensitivity in Section $\mathrm{V}$

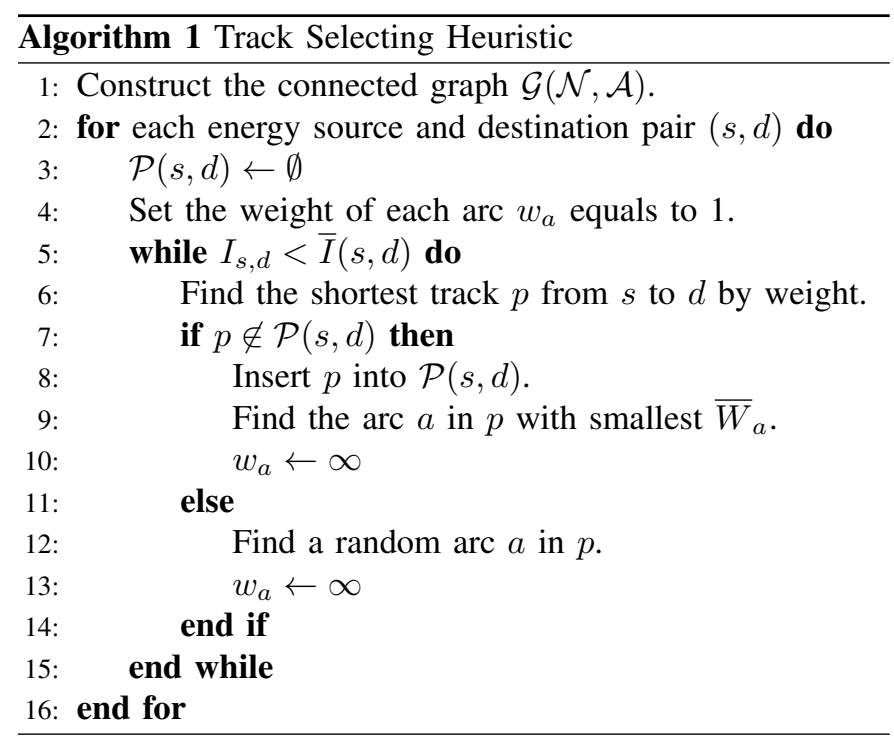

This heuristic introduces a "weight" for each road segment when developing the shortest energy tracks. The weights are employed in the heuristic to determine shortest paths from one energy point to another. For each road segment $a \in \mathcal{A}$, its weight $w_{a}$ can be set to either one or infinity. When it is one, the corresponding road segment can be employed to construct energy tracks. Otherwise, the road segment is excluded in the heuristic. We manipulates the weights of road segments to construct shortest paths for different source-destination pairs, which is described below.

The heuristic starts with the $\operatorname{road}$ network $\mathcal{G}(\mathcal{N}, \mathcal{A})$. For each pair of energy source $s$ and destination $d$, we decide to determine $\bar{I}(s, d)$ energy tracks with the least amount of charging-discharging cycles. To do this, each arc $a \in \mathcal{A}$ is first assigned with a weight $w_{a}=1$ (Line 4). Then we find the shortest track in $\mathcal{G}(\mathcal{N}, \mathcal{A})$ from $s$ to $d$ using the $w_{a}$ values as the metrics (Line 6). Since each arc has weight one, the track $p$ is the shortest from $s$ to $d$ in terms of $w_{a}$. If $p$ has not been generated before, it is considered as a candidate energy track from $s$ to $d$ (Line 8). We continue to develop the next energy path if the total number of generated paths is smaller than $\bar{I}(s, d)$ (Line 5). In addition, to generate a new track different from $p$, we exclude the arc in $p$ which has the smallest energy transmission capacity by setting its weight to infinity (Lines 9-10). If $p$ is already in $\mathcal{P}(s, d)$, a random arc in $p$ is excluded instead (Lines 12-13). This process repeats until enough energy paths have been developed. After the required number of energy tracks have been developed, they are used in modeling Problem (8).

This design has two advantages. First, the shortest $\bar{I}(s, d)$ tracks between energy sources and destinations are always considered. Such paths are usually involved when we minimize the energy loss. Second, those road segments with small energy transmission capacity are less likely to get overloaded; after the first shortest energy track is developed, its segment with the smallest capacity is not used to construct other energy tracks for the same source-destination pair. These advantages allow us to find an optimal solution while avoiding violating the constraints. The efficacy of the proposed heuristic will be demonstrated in Section $\mathrm{V}$

\section{Performance Evaluation}

We conduct three tests to evaluate the performance of the proposed VEN application and the heuristic. We first compare the performance of the proposed heuristic with the optimal solutions for some small problem instances. Then we examine the energy delivery performance and computational time of the proposed problem with heuristic on various problem sizes. Finally we investigate the impact of charging-discharging energy efficiency.

We randomly generate a collection of VEN of different size for each test. In each network, each energy point is connected to some 2-4 energy points nearby by road segments, whose energy transmission capacity is randomly generated in $[2,4]$ units. We randomly select some energy points as the energy sources and destinations. The energy generations at the energy sources are randomly set in $[5,10]$ units while the demands at the destinations are chosen in $[1,2]$ units ${ }^{2}$ All simulations are performed on a computer with Intel Core-i7 CPU at 3.60 $\mathrm{GHz}$ with $32 \mathrm{~GB}$ RAM. The optimization problem and test code are developed with Python 3.

\section{A. Heuristic Performance}

In this test we examine the effectiveness of the proposed energy track selecting heuristic with small test cases. We generate 20 random cases, each of which has 20 energy points with two energy sources and two energy destinations. For these small test cases, we can enumerate all energy tracks so that we can generate the corresponding optimal solutions for comparison.

\footnotetext{
${ }^{2}$ Demands are generally set smaller than generations due to energy loss This allows us to generate feasible problems for evaluation.
} 
TABLE I

Path Selecting Heuristic Performance Comparison

\begin{tabular}{c|c|c|c|c|r}
\hline \multirow{2}{*}{$I(s, d)$} & \multicolumn{2}{|c|}{ Objective Function Values } & \multicolumn{2}{c|}{ Computational Time } & $\begin{array}{r}\text { Average Number } \\
\text { of Energy Paths }\end{array}$ \\
\cline { 2 - 6 } & Delivery & Loss & Track Construction & Optimization & 7685 \\
\hline All & $9.788 \mathrm{E}+00 \pm 2.864 \mathrm{E}+00$ & $6.414 \mathrm{E}-01 \pm 1.818 \mathrm{E}-01$ & $2.129 \mathrm{E}+00 \pm 2.408 \mathrm{E}+00$ & $4.207 \mathrm{E}-01 \pm 3.443 \mathrm{E}-01$ & 8 \\
2 & $9.517 \mathrm{E}+00 \pm 2.508 \mathrm{E}+00$ & $6.513 \mathrm{E}-01 \pm 2.049 \mathrm{E}-01$ & $1.319 \mathrm{E}-02 \pm 6.190 \mathrm{E}-03$ & $2.293 \mathrm{E}-02 \pm 3.665 \mathrm{E}-03$ & 16 \\
4 & $9.681 \mathrm{E}+00 \pm 2.436 \mathrm{E}+00$ & $6.466 \mathrm{E}-01 \pm 1.975 \mathrm{E}-01$ & $1.358 \mathrm{E}-02 \pm 6.488 \mathrm{E}-03$ & $3.497 \mathrm{E}-02 \pm 5.995 \mathrm{E}-03$ & 2.103 \\
6 & $9.730 \mathrm{E}+00 \pm 2.819 \mathrm{E}+00$ & $6.466 \mathrm{E}-01 \pm 1.975 \mathrm{E}-01$ & $1.973 \mathrm{E}-02 \pm 1.332 \mathrm{E}-03$ & $4.264 \mathrm{E}-02 \pm 8.110 \mathrm{E}-03$ & 32 \\
8 & $9.778 \mathrm{E}+00 \pm 2.838 \mathrm{E}+00$ & $6.414 \mathrm{E}-01 \pm 1.818 \mathrm{E}-01$ & $2.573 \mathrm{E}-02 \pm 1.900 \mathrm{E}-03$ & $4.738 \mathrm{E}-02 \pm 8.587 \mathrm{E}-03$ & 40 \\
10 & $9.784 \mathrm{E}+00 \pm 2.838 \mathrm{E}+00$ & $6.414 \mathrm{E}-01 \pm 1.818 \mathrm{E}-01$ & $3.181 \mathrm{E}-02 \pm 2.256 \mathrm{E}-03$ & $5.074 \mathrm{E}-02 \pm 1.065 \mathrm{E}-02$ & 42 \\
\hline
\end{tabular}

TABLE II

ENERgy TRANSMISSION FOR VARIOUS PRoblem SIZES

\begin{tabular}{cccc|c|c|c|c}
\hline \multirow{2}{*}{$|\mathcal{N}|$} & \multirow{2}{*}{$\mathcal{N}^{\mathrm{S}} \mid$} & $\left|\mathcal{N}^{\mathrm{D}}\right|$ & \multirow{I}{*}{$(s, d)$} & \multicolumn{2}{c|}{ Objective Function Values } & \multicolumn{2}{c}{ Computational Time } \\
\cline { 5 - 9 } & & 2 & 2 & $9.517 \mathrm{E}+00 \pm 2.508 \mathrm{E}+00$ & $6.513 \mathrm{E}-01 \pm 2.049 \mathrm{E}-01$ & $1.319 \mathrm{E}-02 \pm 6.190 \mathrm{E}-03$ & $2.293 \mathrm{E}-02 \pm 3.665 \mathrm{E}-03$ \\
20 & 2 & 2 & 4 & $9.681 \mathrm{E}+00 \pm 2.436 \mathrm{E}+00$ & $6.466 \mathrm{E}-01 \pm 1.975 \mathrm{E}-01$ & $1.358 \mathrm{E}-02 \pm 6.488 \mathrm{E}-03$ & $3.497 \mathrm{E}-02 \pm 5.995 \mathrm{E}-03$ \\
& 4 & 2 & 2 & $1.206 \mathrm{E}+01 \pm 3.407 \mathrm{E}+00$ & $4.840 \mathrm{E}-01 \pm 1.606 \mathrm{E}-01$ & $1.463 \mathrm{E}-02 \pm 7.016 \mathrm{E}-03$ & $3.418 \mathrm{E}-02 \pm 3.780 \mathrm{E}-03$ \\
& 4 & 2 & 4 & $1.315 \mathrm{E}+01 \pm 3.417 \mathrm{E}+00$ & $5.296 \mathrm{E}-01 \pm 1.430 \mathrm{E}-01$ & $1.534 \mathrm{E}-02 \pm 8.714 \mathrm{E}-03$ & $4.919 \mathrm{E}-02 \pm 7.085 \mathrm{E}-03$ \\
\hline \multirow{4}{*}{50} & 5 & 5 & 5 & $2.291 \mathrm{E}+01 \pm 5.018 \mathrm{E}+00$ & $1.877 \mathrm{E}+00 \pm 5.197 \mathrm{E}-01$ & $8.247 \mathrm{E}-02 \pm 3.217 \mathrm{E}-02$ & $1.232 \mathrm{E}-01 \pm 1.442 \mathrm{E}-02$ \\
& 5 & 5 & 10 & $2.363 \mathrm{E}+01 \pm 3.337 \mathrm{E}+00$ & $1.736 \mathrm{E}+00 \pm 4.427 \mathrm{E}-01$ & $1.293 \mathrm{E}-01 \pm 4.848 \mathrm{E}-02$ & $1.571 \mathrm{E}-01 \pm 1.969 \mathrm{E}-02$ \\
& 10 & 5 & 5 & $3.062 \mathrm{E}+01 \pm 7.191 \mathrm{E}+00$ & $1.498 \mathrm{E}+00 \pm 4.317 \mathrm{E}-01$ & $1.266 \mathrm{E}-01 \pm 3.571 \mathrm{E}-02$ & $1.811 \mathrm{E}-01 \pm 1.277 \mathrm{E}-02$ \\
& 10 & 5 & 10 & $3.072 \mathrm{E}+01 \pm 6.194 \mathrm{E}+00$ & $1.574 \mathrm{E}+00 \pm 4.248 \mathrm{E}-01$ & $2.195 \mathrm{E}-01 \pm 1.137 \mathrm{E}-01$ & $1.931 \mathrm{E}-01 \pm 2.003 \mathrm{E}-02$ \\
\hline \multirow{4}{*}{100} & 10 & 10 & 10 & $4.452 \mathrm{E}+01 \pm 4.831 \mathrm{E}+00$ & $3.634 \mathrm{E}+00 \pm 5.306 \mathrm{E}-01$ & $1.435 \mathrm{E}+00 \pm 4.686 \mathrm{E}-01$ & $3.386 \mathrm{E}-01 \pm 2.425 \mathrm{E}-02$ \\
& 10 & 10 & 20 & $4.668 \mathrm{E}+01 \pm 4.767 \mathrm{E}+00$ & $3.375 \mathrm{E}+00 \pm 8.358 \mathrm{E}-01$ & $2.797 \mathrm{E}+00 \pm 1.044 \mathrm{E}+00$ & $3.608 \mathrm{E}-01 \pm 3.589 \mathrm{E}-02$ \\
& 20 & 10 & 10 & $6.647 \mathrm{E}+01 \pm 1.235 \mathrm{E}+01$ & $2.942 \mathrm{E}+00 \pm 6.356 \mathrm{E}-01$ & $2.757 \mathrm{E}+00 \pm 1.307 \mathrm{E}+00$ & $4.301 \mathrm{E}-01 \pm 2.819 \mathrm{E}-02$ \\
& 20 & 10 & 20 & $7.282 \mathrm{E}+01 \pm 1.155 \mathrm{E}+01$ & $2.665 \mathrm{E}+00 \pm 3.857 \mathrm{E}-01$ & $5.477 \mathrm{E}+00 \pm 2.720 \mathrm{E}+00$ & $4.770 \mathrm{E}-01 \pm 3.278 \mathrm{E}-02$ \\
\hline \multirow{4}{*}{200} & 20 & 20 & 20 & $9.022 \mathrm{E}+01 \pm 8.102 \mathrm{E}+00$ & $7.565 \mathrm{E}+00 \pm 8.709 \mathrm{E}-01$ & $5.304 \mathrm{E}+01 \pm 4.331 \mathrm{E}+01$ & $1.095 \mathrm{E}+00 \pm 1.073 \mathrm{E}-01$ \\
& 20 & 20 & 40 & $9.196 \mathrm{E}+01 \pm 1.070 \mathrm{E}+01$ & $7.588 \mathrm{E}+00 \pm 1.329 \mathrm{E}+00$ & $1.075 \mathrm{E}+02 \pm 6.930 \mathrm{E}+01$ & $1.238 \mathrm{E}+00 \pm 1.276 \mathrm{E}-01$ \\
& 40 & 20 & 20 & $1.338 \mathrm{E}+02 \pm 1.885 \mathrm{E}+01$ & $5.523 \mathrm{E}+00 \pm 7.940 \mathrm{E}-01$ & $1.016 \mathrm{E}+02 \pm 3.475 \mathrm{E}+01$ & $1.686 \mathrm{E}+00 \pm 1.042 \mathrm{E}-01$ \\
& 40 & 20 & 40 & $1.386 \mathrm{E}+02 \pm 2.003 \mathrm{E}+01$ & $5.428 \mathrm{E}+00 \pm 7.590 \mathrm{E}-01$ & $2.043 \mathrm{E}+02 \pm 8.383 \mathrm{E}+01$ & $1.811 \mathrm{E}+00 \pm 1.327 \mathrm{E}-01$ \\
\hline
\end{tabular}

The objective function values and computational times are presented in Table $\mathrm{I}$, in which both average values and standard deviations are given. The first column gives the number of energy paths generated for each source-destination pair by the heuristic, and "All" means that all energy paths are employed representing the optimal results. We can see that the heuristic can effectively reduce both the times needed for generating energy tracks and for solving the problem. Generally 60$150 \times$ speedup can be expected for track construction and 8$20 \times$ speedup is observed for solving (8). In addition, when $\bar{I}(s, d)$ is large enough, we can determine the global optimal with respect to energy loss while the energy delivery part is very close to the optimal. Even when $\bar{I}(s, d)$ is very small (e.g., 2 in Table I), the objective function values are still satisfactory: only around 3\% and $1.5 \%$ worse than the global optimal. This indicates the efficacy of the proposed heuristic in reducing computational time while generating high-quality energy tracks.

\section{B. Energy Delivery Performance}

In this test, we examine the energy delivery performance of the proposed problem with various problem sizes. We generate test cases with $|\mathcal{N}| \in[20,50,100,200]$. In each case, either $10 \%$ or $20 \%$ of the energy points are energy sources while $10 \%$ are energy destinations. For those instances with more than 20 energy points, we cannot enumerate all possible energy tracks in reasonable time and thus their optimal solutions are intractable. Hence in this test, we can only employ the proposed heuristic to strategically select some energy tracks for the optimization. For each case, we consider two values of $\bar{I}(s, d): 0.1 \times|\mathcal{N}|$ and $0.2 \times|\mathcal{N}|] .20$ random VENs are generated with each combination of the parameters and thus total 320 random cases are generated. The energy efficiency $H$ is set to 0.9. The results, in terms of the average and standard deviation, from solving (8) are presented in Table II

The results clearly demonstrate that both energy delivery and energy loss increases with the problem size. Increasing $\left|\mathcal{N}^{S}\right|$ and $\left|\mathcal{N}^{D}\right|$ lead to larger energy transmission and higher energy loss. Moreover, more energy sources result in significantly higher deliverable amount of energy due to the increased volume of total energy supply. This incurs smaller energy loss because the energy destinations have more sources to be matched with and the ones constituting better energy efficiency are preferred. Furthermore, the number of energy tracks $\bar{I}(s, d)$ slightly increases the energy delivery with no clear impact on the energy loss. This is because that each energy track has its own energy transmission capacity. More energy tracks can increase the total transmission capacity, which relaxes Constraint (5) when solving the problem.

In terms of computational times, it is clear that constructing energy tracks consumes much more time than solving the optimization problem (8). In addition, the time on track construction is roughly proportional to the number of energy tracks required, i.e., $\left|\mathcal{N}^{\mathrm{S}}\right| \times\left|\mathcal{N}^{\mathrm{D}}\right| \times \bar{I}(s, d)$, when the problem size is large (say $\mathcal{N} \geq 50$ ). This linear relationship is not evident for $\mathcal{N}=20$ because the overheads for initializing 


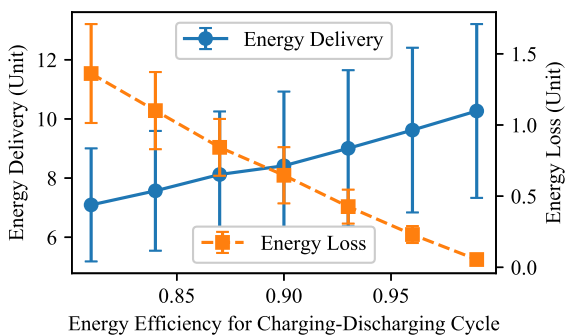

(a) Small case.

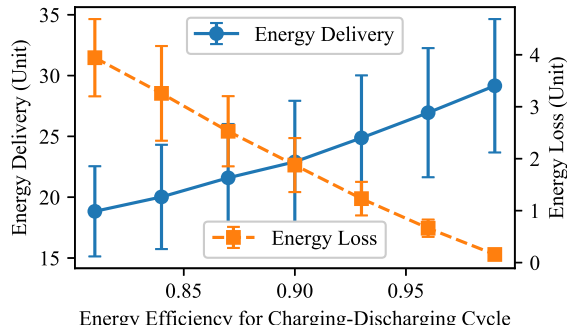

(b) Medium case.

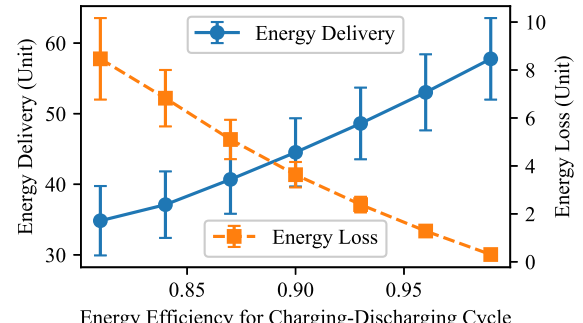

(c) Large case.

Fig. 4. Impact of charging-discharging efficiency on the optimization objectives.

the test code and constructing the road network (Line 1 in Algorithm 11 become significant.

\section{Charging-Discharging Energy Efficiency}

Here we investigate the influence of chargingdischarging efficiency on both the energy transmission and energy loss. Specifically, we set $H \in\{0.81,0.84,0.87,0.90,0.93,0.96,0.99\}$ and consider the small, medium, and large test cases, respectively given by (i) $|\mathcal{N}|=20,\left|\mathcal{N}^{\mathrm{S}}\right|=2,\left|\mathcal{N}^{\mathrm{D}}\right|=2, \bar{I}(s, d)=2$, (ii) $|\mathcal{N}|=50,\left|\mathcal{N}^{\mathrm{S}}\right|=5,\left|\mathcal{N}^{\mathrm{D}}\right|=5, \bar{I}(s, d)=5$, and (iii) $|\mathcal{N}|=100,\left|\mathcal{N}^{\mathrm{S}}\right|=10,\left|\mathcal{N}^{\mathrm{D}}\right|=10, \bar{I}(s, d)=10$. The simulation results are depicted in Fig. 4, where the mean and standard deviation of objective function values are plotted. We can see that the total amount of delivered energy increases with charging-discharging efficiency for all test cases. As the energy transmission capacity is limited, the transmitted energy is either delivered to the energy destinations or lost. So a higher charging-discharging efficiency will lead to a smaller energy loss, which in turn results in better energy delivery performance. Moreover, the energy loss is reduced with charging-discharging efficiency. When minimizing the energy loss, we will only fulfill the minimum energy consumption requirement at the energy destinations. Therefore, a higher charging-discharging efficiency will result in a smaller loss when the delivered energy is unchanged.

\section{CONCLUSION}

With the growing population of EVs, we need to construct more charging stations to accommodate the charging demands of the vehicles. Some charging stations need to be built in remote locations and the renewables are the main energy sources. Due to dissimilar self-generations and local energy demands at different locations, it is beneficial to share the energy among themselves for a sustainable eco-system. VEN has been recently developed and it is capable of transmitting energy over a geographical area by the means of EVs. It is a promising architecture to facilitate energy exchange among these charging stations when we cannot rely too much on the grid. In this paper, we propose a practical solution for energy exchange among some off-grid charging stations through VEN. As a VEN application, we formulate an optimization problem to develop energy exchange plans among the charging stations by maximizing the energy delivery and minimizing the energy loss. Since it is not practical to construct all energy tracks for energy exchange in a large system, we also devise a heuristic to strategically select some energy tracks for optimization modeling. We test the performance of the proposed optimization as well as heuristic with a series of simulations of various problem sizes. The results demonstrate that the proposed design can effectively deliver excessive energy over VEN and the proposed heuristic can drastically reduce the computational time without significantly undermining the performance. This work provides a feasible solution to enable energy sharing among different locations and it can also serve as a guideline for other VEN applications.

\section{REFERENCES}

[1] A. Y. Saber and G. K. Venayagamoorthy, "Plug-in Vehicles and Renewable Energy Sources for Cost and Emission Reductions," IEEE Trans. Ind. Electron., vol. 58, no. 4, pp. 1229-1238, Apr. 2011.

[2] H. Yang, H. Pan, F. Luo, J. Qiu, Y. Deng, M. Lai, and Z. Y. Dong, "Operational Planning of Electric Vehicles for Balancing Wind Power and Load Fluctuations in a Microgrid," IEEE Trans. Sustain. Energy, vol. 8, no. 2, pp. 592-604, Apr. 2017.

[3] H. N. T. Nguyen, C. Zhang, and J. Zhang, "Dynamic Demand Control of Electric Vehicles to Support Power Grid With High Penetration Level of Renewable Energy," IEEE Trans. Transp. Electrification, vol. 2, no. 1, pp. 66-75, Mar. 2016.

[4] W. Shuai, P. Maillé, and A. Pelov, "Charging Electric Vehicles in the Smart City: A Survey of Economy-Driven Approaches," IEEE Trans. Intell. Transp. Syst., vol. 17, no. 8, pp. 2089-2106, Aug. 2016.

[5] E. S. Rigas, S. D. Ramchurn, and N. Bassiliades, "Managing Electric Vehicles in the Smart Grid Using Artificial Intelligence: A Survey," IEEE Trans. Intell. Transp. Syst., vol. 16, no. 4, pp. 1619-1635, Aug. 2015.

[6] A. Y. S. Lam, Y. W. Leung, and X. Chu, "Electric Vehicle Charging Station Placement: Formulation, Complexity, and Solutions," IEEE Trans. Smart Grid, vol. 5, no. 6, pp. 2846-2856, Nov. 2014.

[7] P. J. Tulpule, V. Marano, S. Yurkovich, and G. Rizzoni, "Economic and environmental impacts of a PV powered workplace parking garage charging station," Applied Energy, vol. 108, pp. 323-332, Aug. 2013.

[8] S. Z. Hassan, T. Kamal, S. Mumtaz, and L. Khan, "A Road to Wind Based PHEVs Smart Charging Station," in 2015 13th International Conference on Frontiers of Information Technology (FIT), Dec. 2015, pp. $41-46$.

[9] A. Papavasiliou, S. S. Oren, and R. P. O'Neill, "Reserve Requirements for Wind Power Integration: A Scenario-Based Stochastic Programming Framework," IEEE Trans. Power Syst., vol. 26, no. 4, pp. 2197-2206, Nov. 2011.

[10] A. Y. S. Lam, K. C. Leung, and V. O. K. Li, "Vehicular Energy Network," IEEE Trans. Transp. Electrification, in press. 
[11] S. Jeong, Y. J. Jang, and D. Kum, "Economic Analysis of the Dynamic Charging Electric Vehicle," IEEE Trans. Power Electron., vol. 30, no. 11, pp. 6368-6377, Nov. 2015.

[12] A. Y. S. Lam and J. J. Q. Yu, "Robust Routing for Vehicular Energy Network Routing," in Proceedings of ACM Workshop on Electric Vehicle Systems, Data and Applications, ser. EV-SYS '17, Hong Kong, May 2017.

[13] A. Y. S. Lam and V. O. K. Li, "Energy Loss Minimization for Vehicular Energy Network Routing," in Proceedings of ACM Workshop on Electric Vehicle Systems, Data and Applications, ser. EV-SYS '16, Waterloo, ON, Canada, Jun. 2016, pp. 4:1-4:6.

[14] —_, "Opportunistic Routing for Vehicular Energy Network," submitted for publication. 